\section{FAMILIAL CAMPTODACTYLY COXA VARA PERICARDITIS SYNDROME MASQUARADING AS JUVUNILE IDIOPATHIC ARTHRITIS}

\section{Shahzadi Sumbal Ghazi', Muhammad Umar Nisar ${ }^{2 \bowtie}$, Samer Sikander ${ }^{3}$, Jai Krishin'}

\section{ABSTRACT}

The camptodactyly-arthropathy-coxa vara-pericarditis syndrome (CACP) is a rare autosomal recessive disease characterized by congenital or early-onset camptodactyly, childhood-onset non-inflammatory arthropathy associated with synovial hyperplasia, constrictive pericarditis and coxa vara. This rare disease is caused by mutations in PRG4 gene which encodes for lubricin, a protein present in synovial fluid responsible for lubrication.

The case we are reporting is of 9 and half years old boy who presented to us in pediatric out patient department with early onset camptodactyly and noninflammatory arthropathy. Synovial biopsy shows synovial hyperplasia with numerous CD68 positive multinucleated giant cells. He was discharged and physiotherapy was advised with regular follow ups.

KEY WORDS: Coxa Vara (MeSH); Lubricin (MeSH); Joint Diseases (MeSH); Arthropathy (MeSH); Osteoarthritis (MESH); Pericarditis (MeSH); Synovial Membrane $(\mathrm{MeSH})$.

THIS ARTICLE MAY BE CITED AS: Ghazi SS, Nisar MU, Sikander S, Krishin J. Familial camptodactyly coxa vara pericarditis syndrome masquarading as juvunile idiopathic arthritis. Khyber Med Univ J 2018; $10(4): 221-3$.

\section{INTRODUCTION}

7 he camptodactyly-arthropathycoxa vara-pericarditis syndrome (CACP) is a rare entity that is autosomal recessive (MIM 208250) and presents as a triad of camptodactyly, non-inflammatory arthropathy with hyperplasia of synovial membrane and coxa vara.

Patients with CACP usually has a typical presentation of articular features that mimic most common rheumatic disorders. These patients are commonly diagnosed and treated as juvenile idiopathic arthritis (JIA). ${ }^{2}$

Joints of patients with CACP are normal at birth but as the time passes, they experience a non-inflammatory hyperplasia of synoviocytes resulting into camptodactyly and arthropathy that leads to premature joint contracture. ${ }^{3}$ Coxa vara is progressive and occurs in $50-90 \%$ of patients.

We are presenting a rare case of CACP in a 9.5 years old male who was initially misdiagnosed as juvenile idiopathic arthritis (JIA) and being treated with methotrexate and folic acid. Not responding to the above treatment, child developed progressive camptodactyly of thumb. Child was admitted and investigated and diagnosed to have CACP syndrome.

\section{CASE REPORT}

A 9.5 years male child was admitted with chief complaints of fingers and toes deformity in May, 2015. In May 2013 child was diagnosed as a case of pauciarticular JIA for which methotrexate and folic acid started. He presented to our outpatient department (OPD) due to bilateral knee joint swelling and deformity of fingers which was not responding to treatment. Camptodactyly of thumb was observed soon after birth by the mother that was progressive. We decided to admit the patient for workup and revise the diagnosis. Child had a blood pressure (BP) of $105 / 70 \mathrm{mmHg}$, heart-beat of $88 /$ minute, temperature
I Department of Pediatric Medicine, Children Hospital, Pakistan Institute of Medical Sciences (PIMS), Islamabad, Pakistan

2 Department of Pediatric Surgery, Children Hospital, Pakistan Institute of Medical Sciences (PIMS), Islamabad, Pakistan Email凶:umarnisar222@gmail.com

3 Pakistan Atomic Energy Commission (PAEC) General Hospital, Islamabad, Pakistan

Date Submitted: December 20,2017 Date Revised: October 27, 2018 Date Accepted: October 29, 2018

$98.4^{\circ} \mathrm{F}$ and respiratory rate (RR) of 26/minute. Swelling of bilateral (B/L) knee joint was observed. There was no evidence of fever, rash and lymphadenopathy. Another younger brother has also started to develop similar complaints.

Laboratory findings revealed hemoglobin (Hb) $1 \mathrm{l} .4 \mathrm{~g} / \mathrm{dl}$, total leukocytes count (TLC) 9.9 with $40.5 \%$ neutrophils and $45.6 \%$ lymphocytes, platelets were 198,000 , erythrocyte sedimentation rate (ESR) $9 \mathrm{mmHg}$, C-reactive protein (CRP) negative, rheumatoid arthritis (RA) factor and antinuclear antibody (ANA) factor negative, serum electrolytes $(\mathrm{Na}=14 \mathrm{Immol} / \mathrm{L}$, $\mathrm{K}=3.9 \mathrm{mmol} / \mathrm{L}, \mathrm{Cl}=1.2 \mathrm{mmol} / \mathrm{L})$, urea was $27 \mathrm{mg} / \mathrm{dl}$ and creatinine was $0.56 \mathrm{mg} / \mathrm{dl}$. Chest X-ray was normal. Plain radiograph of hands showed flexion deformity of proximal interphalangeal (PIP) joints of fingers and thumb of both hands (Figure I).

MRI of knee joint showed thin rim like enhancement of fluid filled bursa (Figure 2). Synovial biopsy done and report shows synovial proliferation with numerous CD 68 positive multinucleated giant cells consistent with CACP. $\mathrm{He}$ was diagnosed to have CACP. Orthopedic consultation was taken for splint application and z-plasty after the diagnosis. Echocardiography was done which was normal. He was discharged after advising physiotherapy and was further advised for regular follow up to check for development of pericarditis.

\section{DISCUSSION}

The camptodactyly-arthropathy-coxa vara-pericarditis syndrome (CACP) is a rare autosomal recessive disorder caused by mutation in PRG4 gene. 


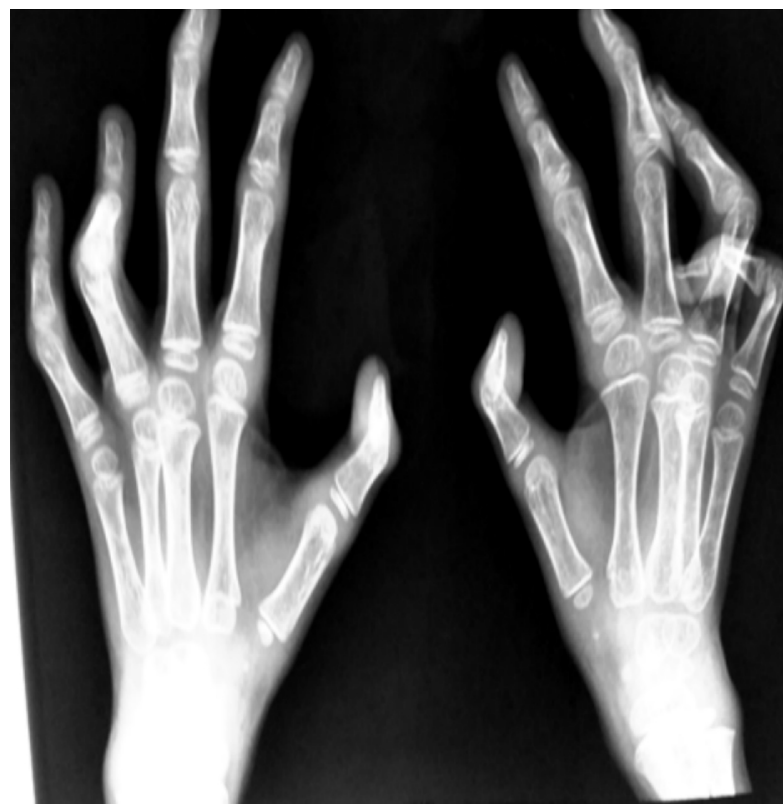

Figure I: X-ray hands showing bilateral camptodactyly

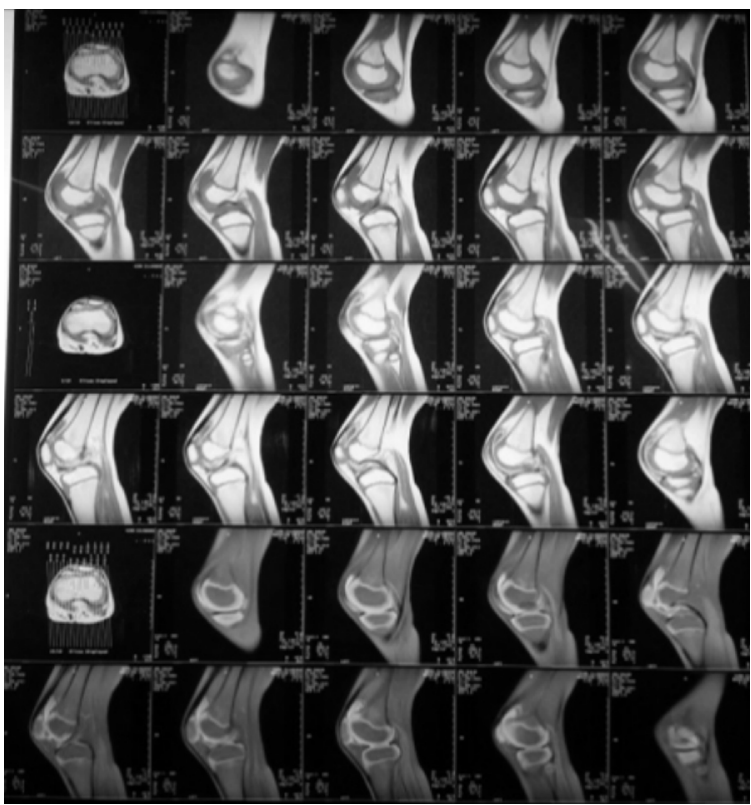

Figure 2: MRI Knee joint showing rim like enhancement of filled bursa
Fifteen (15) mutations in PRG4 gene responsible for causing CACP have been listed in Human Genome Mutation Database (www.hgmd.cf.ac.uk). ${ }^{4}$ PRG4 gene encodes for lubricin, a large protein present in large amount in synovial fluid of joints. It is secreted by joint fibroblasts and chondrocytes and serves as lubricant for boundary and protects articular cartilage form friction induced injury. Lubricin is found in many other parts of body such as on tendon surface, pleural and pericardial cavities, liver, kidney and muscles. In pleural and pericardial cavities lubricin has likely anti-adhesive function. In humans, absence of this important protein is mainly responsible for camptodactylyarthropathy-coxa vara-pericarditis syndrome (CACP). ${ }^{5}$ Consequently, lack of lubricin will lead to synovial hyperplasia, which can have deleterious effects on joint cartilage by inhibiting nutrient and waste exchange. Tissue remodeling and tendon sheath calcification may result into camptodactyly.'

Clinically child with CACP presents with non-traumatic fixed flexion deformity of PIP joints of fingers (camptodactyly), which may be congenital or acquired and usually bilateral resulting into contracture. Usually child complains of swelling, pain and limited range of motion in large joints such as elbows, hip, knee and ankle (arthopathy). Non- inflammatory constrictive pericarditis is present in upto 30 percent cases of CACP published so far and may be mild in nature and is self limiting. ${ }^{6}$ Coxa vara, in which the angle between shaft and head of femur is decreased to less than 120 degrees, results in shortening of affected limb and is present in 50-90\% of the cases, progresses gradually over time.'

Diagnosing patients with CACP syndrome is very important as they mostly present with sign and symptoms which mimic common rheumatic conditions such as JIA and are treated for these, as it was in our case, being treated with methotrexate. ${ }^{2,4}$ Children with CACP have swollen joints that are cool, refractory to anti-inflammatory therapy. Synovial fluid analysis is negative for inflammation. Histopathological findings of synovial tissue shows marked synovial hyperplasia with no evidence of inflammation while hyperplasia of synovium is associated with chronic inflammation in case of rheumatoid arthritis. MRI is useful in differentiating CACP from other connective tissue disorders in children like JIA. MRI of the involved joint will show rim like enhancement of fluid filled bursa on TI weighted image before and after contrast enhancement. ${ }^{6}$ Echocardiography can be done for diagnosis of pericarditis. $^{7}$
Overall prognosis of CACP syndrome is good and most of the patients do not encounter serious problems.

Camptodactyly can be managed conservatively by splinting and physiotherapy but sometimes tenolysis or synovectomy may be necessary. Various new techniques have been tried for synovectomy but without any satisfactory improvement in the treatment of CACP. Al-Mayouf SM, et al. tried Yttrium-90 radiosynovectomy in patients of CACP syndrome but they concluded no beneficial therapeutic effect of their treatment of arthropathy of CACP syndrome. ${ }^{2}$ Pericarditis is usually mild and self limiting but in life threatening cases pericardiocentesis may be necessary.

\section{REFERENCES}

I. Peters B, Schuurs-Hoeijmakers JH, Fuijkschot J, Reimer A, van der Flier M, Lugtenberg D, et al. Protein-losing enteropathy in camptodactylyarthropathy-coxa vara-pericarditis (CACP) syndrome. Pediatr Rheumatol Online J 2016;14(I):32. DOI: I0.I I86/s I 2969-0| 6-0093-5.

2. Al-Mayouf SM, Almutairi N, Alismail K. The Efficacy of Yttrium-90 Radiosynovectomy in Patients with Camptodactyly-Arthropathy-Coxa Vara-Pericarditis Syndrome. Mol 
Imaging Radionucl Ther 2017;26(I):33-7. DOI: 10.4274/ mirt.29484.

3. Ciullini Mannurita S, Vignoli $M$, Bianchi L, Kondi A, Gerloni V, Breda $L$, et al. CACP syndrome: identification of five novel mutations and of the first case of UPD in the largest European cohort. Eur J Hum Genet 20I4;22(2):|97-20I. DOI: 10.1038/ejhg.2013.123.

4. Patil DV, Phadke MS, Pahwa JS, Dalal
$A B$. Brothers with constrictive pericarditis - A novel mutation in a rare disease. Indian Heart J 2016;68 Suppl 2:S284-S7. DOI: 10.1016/j.ihj.2016.03.020.

5. Ai M, Cui Y, Sy MS, Lee DM, Zhang LX, Larson KM, et al. Anti-lubricin monoclonal antibodies created using lubricin-knockout mice immunodetect lubricin in several species and in patients with healthy and diseased joints. PLoS One 20I5;IO(2):e0II6237. DOI: |0.137|/journal.pone.0| |6237.

6. Choi BR, Lim YH, Joo KB, Paik SS, Kim NS, Lee JK, et al. Camptodactyly, arthropathy, coxa vara, pericarditis (CACP)syndrome: a case report. J Korean Med Sci 2004; 19(6):907-10.

7. Tapar M, Eyileten Z, Kasýmzade F, Uçar T, Kendirli T, Uysalel A. Camptodactyly-arthropathy-coxa vara-pericarditis (CACP)syndrome. Turk J Pediatr 20 I 4;56(6):684-6.

\section{AUTHORS' CONTRIBUTIONS}

Following authors have made substantial contributions to the manuscript as under:

SSG: Concept \& study design, drafting the manuscript, final approval of the version to be published.

MUN: Drafting the manuscript, critical review, final approval of the version to be published.

SS \& JK: Critical review, final approval of the version to be published.

Authors agree to be accountable for all aspects of the work in ensuring that questions related to the accuracy or integrity of any part of the work are appropriately investigated and resolved.

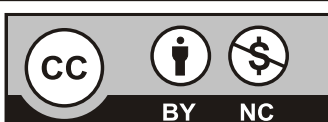

This is an Open Access article distributed under the terms of the Creative Commons Attribution-NonCommercial 2.0 Generic License.
KMUJ web address: www.kmuj.kmu.edu.pk

Email address: kmuj@kmu.edu.pk 